\title{
Artificial Neural Networks for Predicting Customer Expenses
}

R. Rada

B. Ruseti

N.Baçi

M.Karçanaj

Doi:10.5901/jesr.2016.v6n1p172

\begin{abstract}
Artificial Neural Network is becoming more and more pupular in many business fields. Decision is a critical process in business and ANN with its techniques is playing a key role in this point. In this paper we will treat the use of ANN techniques in business, especially integrating it with a CRM (Customer Relationship Management) database.Using ANN techniques we aim to discover the group which spends more in mobile communication. The technique discussed in this article will help Albanian mobile communication companies to find the most profitable group of customers.
\end{abstract}

Keywords: Artificial Neural Networks, Customer Relationship Management (CRM), Data Mining, Multilayer Perceptron, Back Propagation, Activation Function.

\section{Introduction}

Businesses operate based on selling certain products to certain customers. Finding the target group that is more interested on a certain product is the main task of a company. Even though there exists a large number of predictive methods, Artificial Neural Network continues to remain the most efficient one. [Paolo Valigi, Vidas Gulbinas, Rainer Westphal, Khaled Mohamed Almhdi And Rainer Reuter.Classification with Artificial Neural Networks And Support Vector Machines:] .

Usage of such techniques is quite large in many countries. Such a technique doesn't exist in Albania, and as alternative way of predictive tools, companies use descriptive statistics.

In this paper we give a general overview of Artificial Neural Network and its techniques, different activation function used by ANN in prediction and at the end we give a case study of a mobile communication customer's database in Albania. A given data set is trained using back-propagation algorithm in a multilayer neural network. After training we use several activation function types to find the one which has the most precise prediction. In the next section we give the definition of ANN and describe some its activation functions.

In section 3 we give a case study and discuss the Artificial Neural Network integration in mobile communication

\section{Artificial Neural Network}

Artificial Neural Network is computational system implemented as an imitation of human biological brain [N.Bhargava, M.Gupta Application of Artificial Neural Networks in Business Applications.].

A neural network consists of several processing elements connected together called neurons. Those neurons connected together form a dense web of interconnections. A neuron has two main duties. One is that is calculates the output which is send to the other neuron and becomes input of this. The neuron applies a transfer function which determines its output value. Second duty of a neuron is to update the weight (local memory) of its connection.

Neural Networks are organized into layers. One network may have many layers but at least two, input layer and the output layer. The other layers are called inner or hidden layers.

The output of a neuron is computed as the following:

All the inputs multiplied with their respective weight ate added together, and the gained value is put in the activation function. Activation function gives the output of the neuron. 


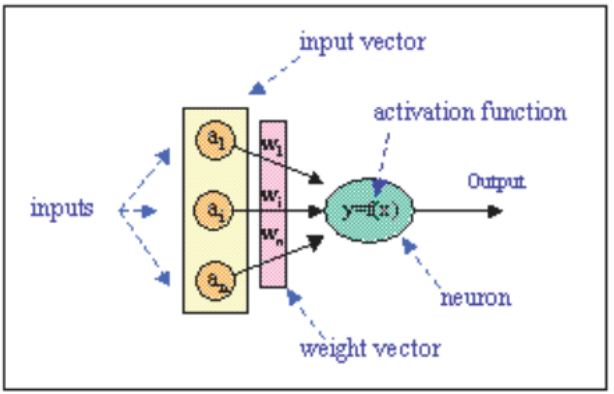

Figure 1: An artificial neuron [Goodchild, Longley.Artificial Neural Networks (ANN)]

\subsection{The activation function}

Activation function has a major role in precision of decision for a neuron. The most primitive activation function is binary, which determines if the neuron should fire or not. In order to get a liked result, a large number of neuron is needed.

In this article we will consider some more sophisticated activation function.

Unipolar sigmoid function $g(x)=\frac{1}{1+e^{-x}}$

Bipolar sigmoid function $g(x)=\frac{1-e^{-x}}{1+e^{-x}}$

Hyperbolic tangent function

$$
\tanh (x)=\frac{\sinh (x)}{\cosh (x)}=\frac{e^{x}-e^{-x}}{e^{x}+e^{-x}}
$$

Conic Section Function

$$
f(x)=\sum_{i=1}^{N+1}\left(a_{i}-c_{i}\right) w_{i}-\cos _{i}\left(\left\|a-c_{i}\right\|\right)
$$

\subsection{Learning methods and ANN's architecture}

Artificial Neural Network is based on the model of human brain which learns from the experience and then performs testing over the training. It is composed of two main phases which are training and testing. The goal of the training phase is to find the training parameters called weights [Goodchild, Longley.Artificial Neural Networks (ANN)].

Artificial network uses two data sets during the whole process, which are training and testing sets. The network uses training set to adjust and train weights in order to produce target outputs. Testing set is used to evaluate the trained artificial neural network. In order to keep independence the training and testing sets are not the same.

One of the most well-known artificial neural networks is Multilayer Perceptron (MLP) which is used for various machine learning tasks. Neuron is the most basic component of MLP. In this network neurons are organized in layers and neurons of every layer are connected with weighted edges. A multilayer perceptron consists of at least three layers, input, hidden and the output layer. Input layer receives firstly the stimulus and then sends it to the next layer which is of type hidden. Hidden layer computes the weighted sum which is processed by an activation function [P.C. Pandey. Multilayer Perceptron In Damage Detection Of Bridge Structures]. The result of it is propagated to the next layer as input. [Cho-Pu Lin, Yann-Haur Huang, Chien-Ting Chen, Hsing-Yun Chang.Crm Implementation Strategy In Taiwan's Banking Industry]

\section{Case Study}

In this section we will give a general overview of the application of Artificial Neural Network on the business. Our case study is related to the application of ANN in mobile communication by analyzing a customer's database. Every customer is characterized by several personal information. According to such given information we will try to connect them with the amount of money spend on communication.

The application consists on finding the activation function that best predicts the group who spends more on mobile communication. A dataset of around 930 customers will be taken in consideration in this study. 


\subsection{Methodology}

Although there are many other techniques, we will mention a number of advantages that make Artificial Neural Network to be more efficient: require less formal statistical training, ability to detect complex nonlinear relationships, ability to detect possible interactions between predictor variables and the availability to use one of many training algorithms that it offers. [Velu C. And Kashwan.Pareto Classification Of Data Mining For Customer Relationship]

The database is divided into two datasets, training and testing. Our case study is based on supervised learning and the application is bases on back-propagation algorithm which tries to adjust the weights of neuron pairs in order to receive best prediction. [P.Isakki Alias Devi And Dr.S.P.Rajagopalan.Mining Unstructured Data Using Artificial Neural Network And Fuzzy Inference Systems Model For Customer Relationship Management]

\subsection{Training Data Set}

The data set consists in around 620 records of customers respectively with input and output. Each customer is composed of different variables such as: age, marital status, social status, personal income, family income, number of persons in family and the output variable which are the expenses per month in mobile communication. (Table 2)

\begin{tabular}{|l|c|c|c|c|c|c|c|c|}
\hline ID & Age & marital status & Social status & Personal income & Family income & $\begin{array}{c}\text { Nr. of persons } \\
\text { in family }\end{array}$ & $\begin{array}{c}\text { Nr. of brothers/ } \\
\text { sisters }\end{array}$ & $\begin{array}{c}\text { Monthly } \\
\text { expenses }\end{array}$ \\
\hline 068 & 19 & single & student & 0 & 50,000 & 4 & 2 & 1,500 \\
\hline 189 & 28 & single & employed & 100,000 & 200,000 & 3 & 3 & 5,000 \\
\hline 137 & 20 & single & employed & 10,000 & 50,000 & 4 & 3 & 4,000 \\
\hline 079 & 35 & married & employed & 10,000 & 40,000 & 5 & 7 & 2,500 \\
\hline 452 & 39 & married & employed & 17,000 & 100,000 & 8 & 7 & 2,000 \\
\hline 362 & 21 & single & student & 0 & 83,000 & 4 & 2 & 3,000 \\
\hline 278 & 19 & single & student & 0 & 50,000 & 3 & 4 & 7,500 \\
\hline
\end{tabular}

Table 2: Database sample input

The values of variables for the ones of two value variable domain, we write them as 0 or 1 . The table below shows a version of converted variables to numerical one (Table 3).

After we transform them into numerical values, next step is to normalize them. We use the method min-max standardization. [Staffordshire University. Multilayer Perceptron Tutorial Leonardo Noriega].

\section{Artificial Neural Network Implementation}

ANN algorithm is implemented in Java programming language. We will be describing in detail the steps being taken for the implementation of the algorithm, starting from the receipt of the information, processing them and then analyzing the conclusions.

In the following section are presented the steps being taken for the implementation of the entire algorithm.

Step 1: The transformation of data from Excel into CSV format which will then be used by Java code.

Step 2: Development of section for receiving data from CSV file format and storing them in different structures or objects used Java language as Array or ArrayList.

Step 3: Implementation of ANN Algorithm

\subsection{Variables And Data Processing}

In order to process such customer variables we should convert them into numerical values. The numerical variables have been normalized so the while processing them we will not get big numbers and the weights are found easier. [Werner Peeters.An Overview of Fuzzy Control Theory] 
Table 3: Database sample input with numerical variables.

\begin{tabular}{|c|c|c|c|c|c|c|c|c|}
\hline ID & Age & $\begin{array}{c}\text { marital } \\
\text { status }\end{array}$ & $\begin{array}{c}\text { Social } \\
\text { status }\end{array}$ & $\begin{array}{c}\text { Personal } \\
\text { income }\end{array}$ & $\begin{array}{c}\text { Family } \\
\text { income }\end{array}$ & $\begin{array}{c}\text { Nr. of persons in } \\
\text { family }\end{array}$ & $\begin{array}{c}\text { Nr. of brothers/ } \\
\text { sisters }\end{array}$ & $\begin{array}{c}\text { Monthly } \\
\text { expenses }\end{array}$ \\
\hline 068 & 19 & 1 & 0.5 & 0 & 50,000 & 4 & 2 & 1,500 \\
\hline 189 & 28 & 1 & 1 & 100,000 & 200,000 & 3 & 3 & 5,000 \\
\hline 137 & 20 & 1 & 1 & 10,000 & 50,000 & 4 & 3 & 4,000 \\
\hline 079 & 35 & 0 & 1 & 10,000 & 40,000 & 5 & 7 & 2,500 \\
\hline 452 & 39 & 0 & 1 & 17,000 & 100,000 & 8 & 7 & 2,000 \\
\hline 362 & 21 & 1 & 0.5 & 0 & 83,000 & 4 & 2 & 3,000 \\
\hline 278 & 19 & 1 & 0.5 & 0 & 50,000 & 3 & 4 & 7,500 \\
\hline
\end{tabular}

Initial data are stored in a file in XLS format. To make the data reading easier by the application, the file is converted into CSV format. Such format separates the cell values by semi-colon ';,'.

The table (Table 4) below show two states of date the first is in excel forma while the other is in CSV.

Table 4: CSV format of converted data.

\author{
Ina;Sina;19;0.2;0;0;50000;4;2;1500 \\ Miri ;Hoxha;28;0.2;0.5;100000;200000;3;3;5000 \\ Refit;Sulmina;20;0.2;0.5;10000;50000;4;3;4000 \\ Blerta;Teta;35;0.4;0.5;10000;40000;5;7;2500
}

\title{
4.2 Implementation
}

The application is developed in Java programing language. Considering the wide usage and extensive libraries that Java offers, we decided to use this language for the implementation of the program.

To execute the application a version of Java is needed to be installed. As development environment (IDE) we have used Eclipse. Being easy and portable is the reason of using of this platform.

The application consists of several classes as the following:

- Customer.java

- ReadCSV.java

- NeuralNetwork.java

- Neuron.java

- Connection.java

\subsection{Customer Class}

Customer class is created temporary store a customer's data. This class contains all the variables needed to store the data such as name, surname, age, marital status etc.

\subsection{ReadCSV Class}

ReadCSV class is used to get information from CSV format files and storing them in lists that are provided by Java. This class makes validation of data takes from CSV file and then saves each customer in the Customer class objects. All objects are stored in a list of consumers of type customer. This class also calculates the min-max values of the age, personal income and household income. These values are used later in the standardization of data.

ReadCSV class contains two methods:

- getMaxMin ()

- getResources ()

GetMaxMin methods finds the minimum and maximum values for age, personal income and household income. The method takes a String parameter with the address of the file for which the action will occur. 
GetResources methods is implemented to obtain all customers stored in CSV format file by saving them in a list of type Customer. This method works as the following:

1. Opens file

2. Read a row

3. Validated row

4. Establish a Customer object class and given variables values

5. The object is added to the list

6 . For all rows go to step 2

Once the CustomerObj has been filled with the appropriate values, it is added to the CustomerList. This process continues for all consumers and at the end the list will be send to the class where ANN algorithm is implemented.

\subsection{NeuralNetwork Class}

This class is implemented the logic of ANN algorithm. NeuralNeteork.java class communicates with ReadCSV.java to access the list of consumers. There are several techniques implemented for the activation of neurons as following:

1. Unipolar sigmoid

2. Bipolar sigmoid

3. Hyperbolic tangent

4. Conic section

The application works with three Layers (layer) which are: input, hidden and output. Method run is the method which allows the start of the execution of the activation and Backpropagation. This method takes two parameters, a maximum number of tests and one more parameter which is the error value. For each customer algorithm works as described below:

1. For each step up to the given maximum number:

a. Call setlnput method sets the input vector layer with a customer data

b. Calling the method activate and output is calculated

c. on the basis of output and error method Backpropagation is called

2. Stop

3. Activate the neurons with test data

4. For each data vector calculate the output and successful threshold crossing.

Back-Propagation algorithm requires function derivation in order to calculate the updating of the weights. For this reason partialDerivation method was created where derivation of the activation functions are implemented.

We run the process of adjusting the weights by activation neurons and applying back-propagation until the maximum number of steps is reached.

\subsection{Neuron Class}

Neuron is a class that implements a neuron with its features presented in algorithm. This class contains data about neuron. Links are simulated in another class called Connection.

Class Neuron has declared inside an ID, bias, output, in connections.

\subsection{Connection Class}

This class contains all the connections between a neuron others. This makes the calculation of the output very easy because there are stored actual and previous connection weights between two neurons. The connection weights are updated by back-propagation method in the neural network class.

\section{Results}

After training the next process is to test the remained 310 record of customers. The predictions are made using a numerous ANN models. After the predictions are generated, we compare them with the outcome in the testing dataset. According to the calculation of compared date we get the following table of results. There is given the percentage of the right predictions. 
Table 5: Table of results.

\begin{tabular}{|l|c|}
\hline \multirow{2}{*}{ Function } & Dataset validation \\
\cline { 2 - 2 } & Good classification rate (\%) \\
\hline Unipolar sigmoid & $80.4 \%$ \\
\hline Bipolar sigmoid & $67.8 \%$ \\
\hline Hyperbolic tangent & $82.4 \%$ \\
\hline
\end{tabular}

According to the results of the table we notice that the method that gave the best prediction is the Hyperbolic tangent function.

\section{Conclusion}

The main benefits of a company depend directly on the definition of the target consumer for a certain product. Seeing that traditional methods are not very effective, in this article we have treated possibility of integration of data mining techniques in business. We gave a general overview of the Artificial Neural Network in Mobile Communication. According to the received results we saw that this method was more efficient than traditional ones. The data examined are taken from a survey with about 930 records. We trained over 620 consumer and results of testing on the basis of training were very good. According to comparison of results, we noticed that the best network was based on the hyperbolic tangent function which gave accurate results for $82.4 \%$ of test cases.

\section{References}

Classification with Artificial Neural Networks And Support Vector Machines: Paolo Valigi, Vidas Gulbinas, Rainer Westphal, Khaled Mohamed Almhdi And Rainer Reuter.

Application of Artificial Neural Networks in Business Applications N.Bhargava, M.Gupta

Artificial Neural Networks (ANN) Goodchild, Longley.

An Overview of Fuzzy Control Theory, Werner Peeters.

Multilayer Perceptron Tutorial Leonardo Noriega, Staffordshire University.

Multilayer Perceptron In Damage Detection Of Bridge Structures, P.C. Pandey.

Crm Implementation Strategy In Taiwan's Banking Industry, Cho-Pu Lin, Yann-Haur Huang, Chien-Ting Chen, Hsing-Yun Chang.

Pareto Classification Of Data Mining For Customer Relationship, Velu C. And Kashwan.

Mining Unstructured Data Using Artificial Neural Network And Fuzzy Inference Systems Model For Customer Relationship Management P.Isakki Alias Devi And Dr.S.P.Rajagopalan. 